\title{
Acquiring Expert Knowledge on IS Function Design
}

\author{
P. Mantelaers
}

Faculty of Technical Mathematics and Informatics

Delft University of Technology

2600 AJ Delft, The Netherlands

Tel: $\quad+31152784427$

Fax: +31152786632

E-mail:P.A.H.M.Mantelaers@is.twi.tudelft.nl

\begin{abstract}
Reorganizing the IS function can contribute to its efficiency and effectiveness. Management can choose from a large number of organizational options. This leads to uncertainty and a need for decision support. The knowledge of experts in this domain was elicited using think aloud protocols. Next the protocols were analyzed to derive guidelines that can be applied in practice. During the analysis, several techniques were tried. This knowledge acquisition process turned out to be very complex and labor intensive but it also was a rich source of information.
\end{abstract}

\section{INTRODUCTION}

Every organization in the private and in the public sector ought to (re)consider the organization of its IS function (ISF). From reports in professional periodicals it is shown that, in practice, many organizations reorganize their ISF. Applied research (e.g., Butler Cox 1991) shows that many organizations expect to adapt their ISF in the near future and have (repeatedly) done so. A more fundamental interest in this subject is apparent from articles in scientific journals (e.g., Swanson and Beath 1989) and from conference reports. 
There are several reasons to change the organization's ISF: problems with efficiency or effectiveness, dissatisfaction with the ISF of users/customers within the organization, the desire to follow trendy developments, and reorganization to bring about other changes. A more fundamental development is the fact that an increasing number of organizations are becoming aware of the strategic value of information. A conclusion to be drawn from this is that an organization's ISF deserves more attention. Conversely, thinking about the organization's ISF may increase the insight into the strategic value of information.

With the extension of the number of possible solutions (through the loss of oppressive boundary conditions from the available technology and the informaticians available on the labor market), the organization's ISF problem has not become simpler or less important. Just as in designing information systems, in designing an organization's ISF it is always a matter of more than one solution. In principle, it is possible for various IS tasks to come to independent choices from all possible combinations of (de)centralization and (de)concentration. The answer to the question what would be the best alternative for a certain organization, will depend on specific circumstances.

This paper deals with the research approach I have chosen (Mantelaers 1995) to provide a scientifically justified contribution to answer the following practical question: "How can we design the most appropriate organization of the IS function for an organization in a certain situation?"

The ISF was the subject of the research. Its organization was considered within a certain organization to be defined beforehand. The external interorganizational organization problem was left out of consideration. A priori it was supposed to be known whether activities were executed inside or outside the organization. Another restriction was that only the design of the organization of ISF was taken into consideration and not the transition processes resulting from this. The organization, on behalf of which ISF had to be organized, is supposed to be known and fixed.

Three approaches were used to try to gain insight into an organization's ISF methodologies. First, directives for the organization of ISF were gathered from literature. In doing so, attention has been paid to general organization design theories (e.g., Douma and Schreuder 1992), to (methodologies for) information strategy planning (e.g., Turner et al. 1988), and to research in the organization's ITF field carried out by others (e.g., Bacon 1990). An analysis also has been carried out of the reorganization of ISF as it has, in the past, actually taken place within two organizations. The third and most important approach will be dealt with in this paper: the execution of a knowledge acquisition process.

This is an empirical paper that takes a qualitative approach in studying the design of the ISF. In section 2, the rough idea underlying the research approach will be explained. Section 3 focuses on the knowledge elicitation (selecting a method from the available knowledge elicitation methods), choice of experts and organizations and the setting of the experiments. The knowledge analysis (selection of and experiments 
with several analysis techniques) is covered in section 4 . The results of the acquisition are summarized in section 5. Conclusions are drawn in section 6.

\section{THE RESEARCH APPROACH}

As indicated in Figure 1, there is, on the one hand, the problem of the design of the ISF. In this research, the design process is performed by experts. They collect information on the ISF and on its environment and use their expertise to come up with ideas to change the ISF in such a way that it reaches its goals better than before. On the other hand, there is the research problem: based on information about the ISF design processes by experts and on information from other sources, the researcher wants to draw conclusions (design a methodology) as to the improvement of future design processes.

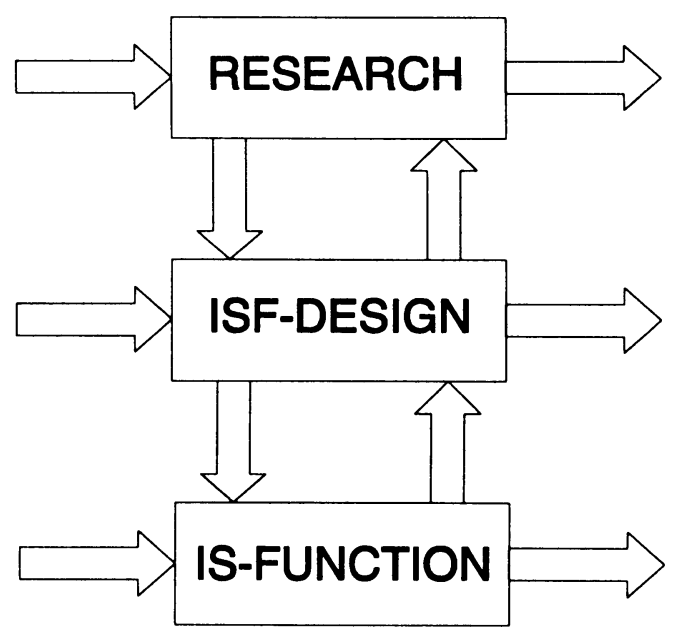

Figure 1 Two Control Problems: ISF Design and Research on ISF Design.

The essence of the research approach concerns the organization of the knowledge acquisition process (sections 3 and 4 deal with details regarding the elicitation and the analysis). The idea behind it is to invite three ISF experts to give their opinion on the way in which they would set up the ISF for three organizations (Rabobank Nederland, Fokker Aircraft and the Ministry of VROM). Each of these nine experiments (cells of the matrix in Figure 2) is first analyzed individually. The results have been compared together. In doing so, mainly similarities per organization (between experts) 
and per expert (between organizations) have been sought. Activities, concepts, and ways of thinking that are found in every experiment have are considered to be elements of a general approach. It is also possible that the analysis leads to general approaches for a certain (type of) organization or for a certain expert. The final result this research aims at is not a normative model that automatically leads to the best ISF, but a set of acceptable statements.

\section{KNOWLEDGE ELICITATION}

During the elicitation step knowledge is extracted from the expert and documented. During the analysis step this verbal data is analyzed, interpreted and modeled. This paragraph describes the knowledge elicitation. Several available methods are described (section 3.1) and from these, one is selected (section 3.2) and applied. The preparation of the experiments is covered in section 3.3 and their execution in section 3.4 .

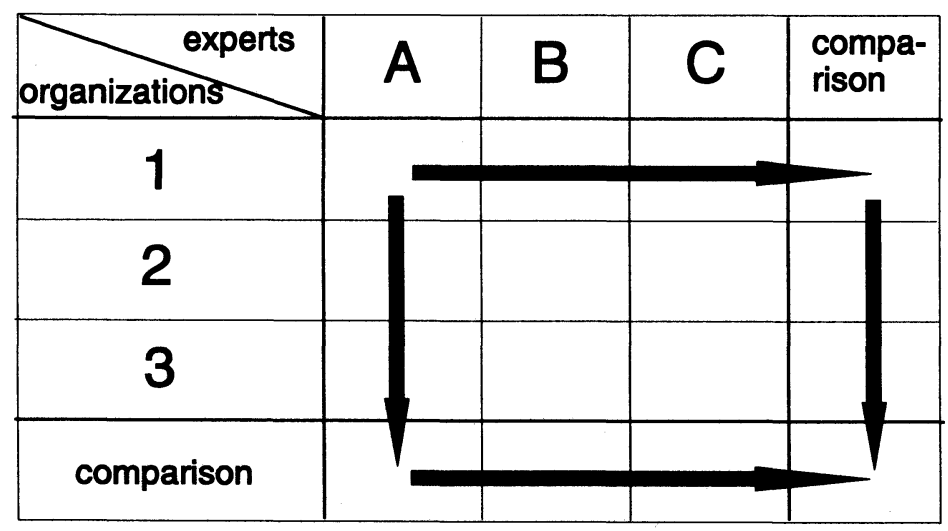

Figure 2 Nine Experiments: Three Experts and Three Companies.

\subsection{Available Elicitation Methods}

Most expert knowledge cannot be observed directly but must be elicited from experts. The following list of most popular methods used in eliciting this knowledge is derived from Wijers (1991). 
Interviewing. Interviewing is the most familiar method. It is widely used because it is relaxed and acceptable. Interviews are recorded question-answer sessions. There are two types of interview: unstructured and structured. Unstructured interviews closely resemble normal conversation. The method provides rough insight into domains. Structured interviews can be compared with interrogations. Knowledge engineers attempt to elicit knowledge about concepts or models by continuously soliciting clarifications, explanations, consequences and justifications. Interviews are effective in rapidly eliciting basic domain structures and when much of the knowledge explicit to experts can be elicited easily. Interviewing is unsuitable for eliciting detailed or poorly accessible domain knowledge and it relies heavily on uncued recall. Interviews especially encourage experts to speculate on and theorize about their cognitive processes.

Think aloud protocols. In these protocols, experts are asked to think aloud (or talk) about every thought and action while working on problems in their field of expertise. This verbalization is recorded and then typed out. The problems may be real or imaginary ones. It is argued that think aloud protocols do not change task performances, although they are slowed down. However, asking experts to explain why they are doing what they do requires them to attempt to access additional knowledge, which disturbs task performance.

Think aloud protocols are particularly suited to eliciting information about the whens and hows of the use of specific knowledge and to abstract the reasoning strategies and task decompositions that follow. Protocol analyses have the disadvantages of being time consuming and that, similar to interviews, knowledge engineers can only capture a series of verbal statements which must be converted to knowledge modules by an interpretative process of sifting, selection and re-representation. Furthermore, in think aloud protocols, knowledge engineers should be aware of the experts' inexperience in self-reporting and of the inaccessibility of proceduralized knowledge.

Think aloud protocols as defined above are known as concurrent protocols. Other forms of think aloud protocols are selective protocols, simulation by teletype and retrospective protocols (i.e., observation and review). Only part of the task is performed in a think aloud way in selective protocols. Simulation by teletype has been used in dialogues where experts could not be provided with completely specified problem statements in advance. The dialogues are held by teletype and both parties are asked to think aloud.

In retrospective protocols, task performances are recorded on audio or video. Reviewing the tape after having solved the problem, the experts are invited to comment on their thoughts and actions. However, retrospective protocols are not so reliable as concurrent verbalizations, because they depend too much on human memory and biases.

Introspection protocols. Introspection involves thinking aloud while solving artificial problems and is, therefore, unlike thinking aloud in problem solving because the process is not based on actual problems. Experts are encouraged to suggest 
feasible solutions, possibly relying on previous experience. Introspection protocols are shorter than think aloud protocols and contain many meta-descriptions and process comments. Introspection is effective in getting experts to sketch their views on the strategies they apply in problem solving, including their justifications.

Observational studies. Observations are similar to think aloud protocols but experts are not expected to think aloud while working. They can be recorded on video and could include telephone conversations. Observations are particularly useful in determining the experts' roles and activities in processes, but poor in establishing the applied reasoning strategies.

\subsection{Method Selection}

The initial idea was to use concurrent think aloud protocols using simulation by teletype as it was applied by Wijers. However, this was unanimously and independently rejected by the experts. They refused to work under such circumstances. The setting (see Figure 3) was considered to be too unrealistic to solve the defined problem. Given the type of problem, eye contact with the interviewees was considered to be indispensable. This puts constraints on the selection of the elicitation method.

From the available methods for knowledge elicitation, a combination of observation and think aloud protocols was chosen. Concurrent protocols and, additionally, retrospective protocols were used, however, in both cases selectively: during the task implementation, not every thought was expressed by the experts and afterwards not everything was explained. This method was completed by unstructured interviews.

In section 3.4, attention will be paid to the setting in which the experiments took place.

\subsection{Preparing the Experiments}

Selection of experts. To be qualified for participation in the research, the experts had to have had many years of experience in the field of the organization of the ISF, to be acknowledged as experts by professionals in the field of study, and to be prepared to reserve at least three times a period of two days for the research without being paid. The experts were told explicitly that this research did not aim at evaluating their individual advice. The advice and the processes preceding it would only be used as a means to come to conclusions about knowledge at a higher level. The top three of the list of acceptable expert-candidates agreed to take part in the experiments.

Selection of companies. With regard to the organizations, a certain spread was desired: both the private and the public sector had to be represented and, within the private sector, both physical and information-related transformation processes had to be involved. This spread was chosen because of the assumption that these distinctions in particular would influence the design of the ISF. Cooperating organizations would get three free-of-charge consultancy reports regarding their ISF by independent experts. However, employees, especially at higher levels in the organizations, had to 


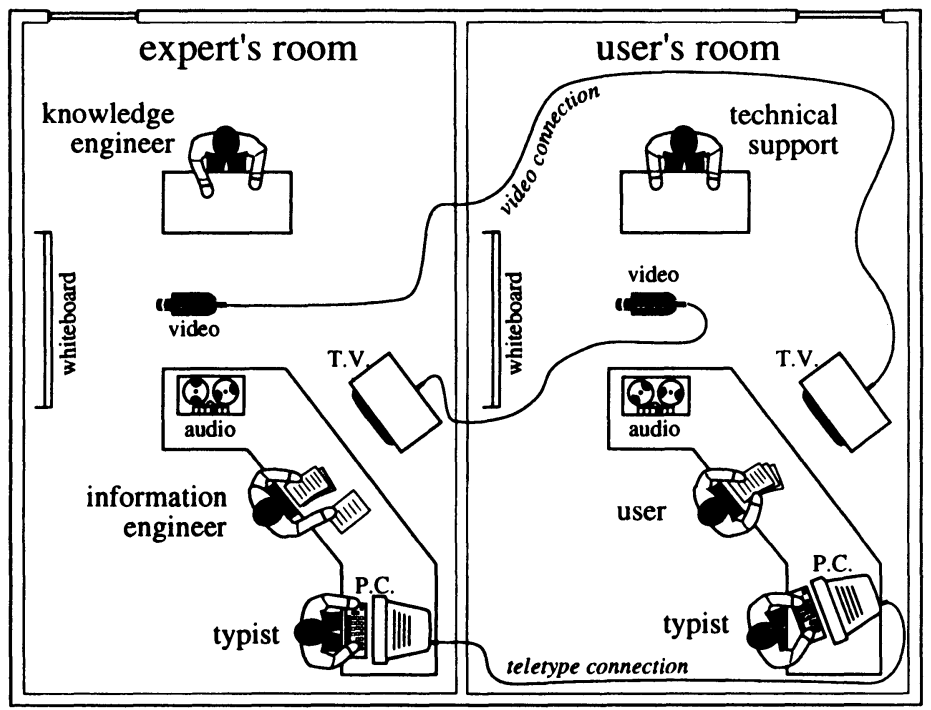

Figure 3 The Initial Idea (Wijers 1991).

be prepared to spend enough time on the project. They also ran the risk that employees not involved would get suspicious. We looked for organizations that recently reorganized their ISF. The experiments could, then, function more or less as an $e x$ post evaluation of their transition.

Task to be fulfilled. Each experiment consisted of carrying out a consulting assignment in the field of ISF by an expert and was allowed to take two days at the maximum, inclusive of the formulation of the advice. In these two days, the expert could, in the presence of the researcher, have a number of interviews with functionaries of the organization in question. The whole experiment was registered on videotapes and audiotapes.

Selection of interviewees. The experts knew what they were expected to do but they did not know the names of the three organizations where they had to fulfill their mission. Informing them about their task was, of course, necessary to get their cooperation and also to offer them the opportunity to determine what functionaries they wanted to interview when performing their consultancy. The description of interviewees could only be done in general terms, due to the fact that the experts did not know the names of the companies. Two days were available for interviews. Taking into account that advice had to be formulated as well, about six interviews could take place in two days. This number was not considered to be a limitation by the experts. In close cooperation between the researcher and representative of each 
company and taking into account the requirements formulated by the experts, it was decided which persons were going to be invited to take part in the interviews. They all received a letter to explain the background of the experiment.

\subsection{Execution of the Experiments}

Information provided. From the research point of view, it was preferred to give the experts as little information as possible. This would prevent them from preparing their work without the researcher being present to record as much of the thinking process as possible. However, the experts had to be told what they were expected to do, otherwise they would not have cooperated and would not be able to determine who they wanted to interview.

The names of the companies were kept secret as long as possible. At the first company the experts only knew its name one hour in advance. At that point, they were given a document with general information about the organization (structure, information systems, number of employees, etc.) written by the researcher. They also received the interview schedule for the two days, including some background information about the interviewees. Due to time constraints, in the later experiments this information had to be provided to them the day before the experiment.

The interviews. This paragraph does not deal with the contents of the interviews but with the setting in which they took place. Not being able to have eye contact with the interviewees was unacceptable for the experts. Nonverbal communication was considered to be so essential in getting used to each other, in making interviewees feel comfortable, in influencing them or in the interpretation of their replies that the setting needed modification. The solution was to use an online video and audio connection between the two rooms. The setting of the experiment that was used for the first three experiments (with a large bank) is represented in Figure 4. A disadvantage was that it was impossible to think aloud during the communication with the interviewee. To compensate for this drawback, a time-out was introduced every twenty minutes by disconnecting the audio and video connection. During the time-out, the expert had the opportunity to think aloud about what happened until then and about the continuation of the interview. Some complementary questions were asked by the researcher. In the interviewee room, a research assistant was present to hear the opinion of the interviewee about the interview and the expert so far. He also asked the interviewee's opinion on differences between this expert and the other experts. These time-out conversations were only recorded on audio. Before starting an interview, the expert and his interviewee had a short opportunity to shake hands.

After the completion of the first three experiments, the setup was evaluated. According to all people involved, the whole process developed rather smoothly. The experts stated that the selected method of communication hardly hindered the communication. In spite of that, it was decided to put the expert and the interviewee in the same room. During time-outs, the expert and the researcher would go to an adjoining room. This new setting, which was applied from then on, is illustrated in Figure 5. 


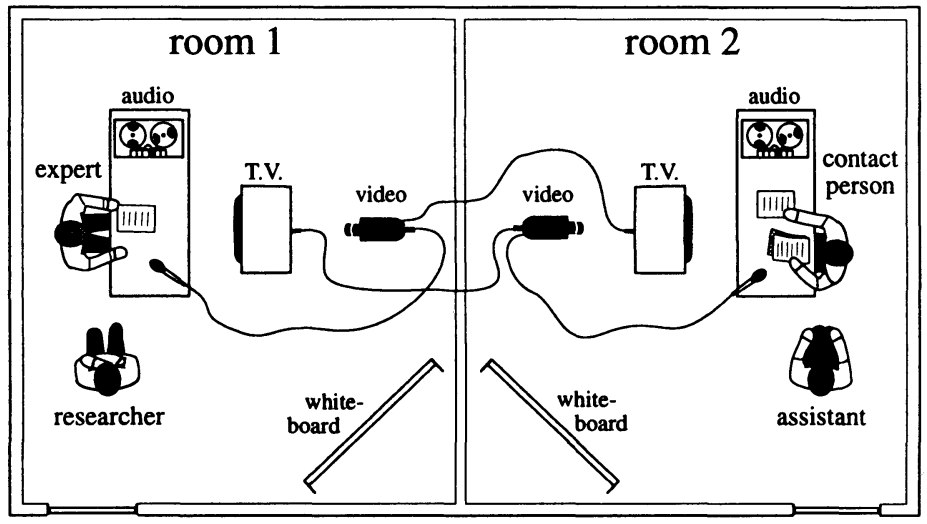

Figure 4 First Interview Setting.

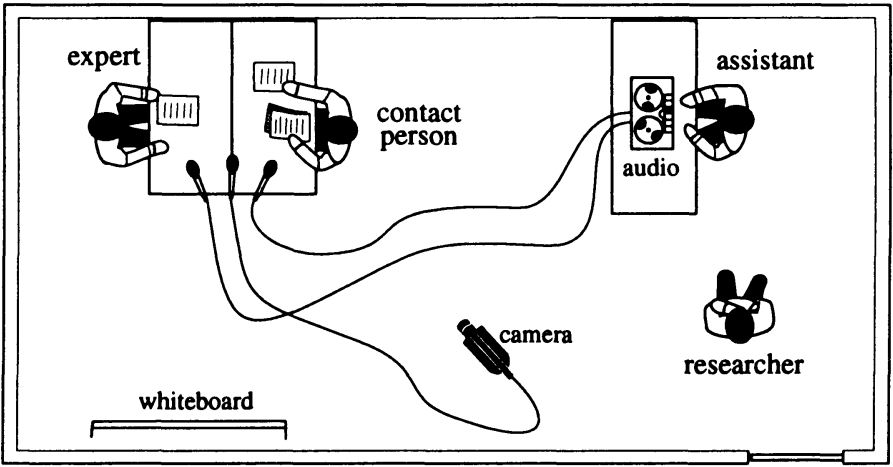

Figure 5 Second Setting.

The most important reason to change the setting was the idea that the clear presence of the video equipment might keep certain interviewees from providing certain information. In the new setting, video recordings were made as well, but the TV screens were no longer necessary and the location of the camera was less emphatic. The interviewee was placed with his back toward the camera. After being explained to the interviewees, the fact that the time-outs took place in another room was accepted by them as unavoidable from the perspective of the research objective.

The advice. Immediately after each experiment the expert was asked to formulate advice as to the IS function of the company involved. The experts used the notes they took during the interviews and their memory to recapitulate the most salient details 
of each interview. This formed another opportunity to register their ideas about important concepts, tasks and information while working on the design of the ISF. It also offered the researcher the opportunity to pose questions about the process. On the basis of the recordings of these conversations, the researcher formulated the advice and asked the expert to correct and complete it if necessary. The advice of the three experts was then presented to each company. They were presented as "a number of ideas" and it was emphasized that these ideas were just a by-product and not the main product of the process.

The transcripts. All conversations were recorded on audio and video tapes; timeout conversations were recorded on audio only. To make this material ready for analysis, all conversations had to be transcribed. This was not an easy task and was very labor intensive. The protocols were checked and corrected by the researcher. Having done this, 3,000 pages with conversations were ready to be analyzed.

\section{KNOWLEDGE ANALYSIS}

\subsection{Introduction}

The protocol transcriptions (on the basis of conversations recorded on audio and video tapes and transcribed literally) formed the starting point for the knowledge analysis. The aim was to isolate all knowledge that might be needed in solving the organization's ISF problem. In analyzing the protocol transcriptions and the representation of the knowledge to be drawn from it, it had to be taken into consideration that each experiment had to be described in such a way that mutual comparison of the results of the nine experiments was feasible. Next to that, the description ought to make it possible for the results to be used in practice by those who were considered to take decisions or make proposals about the organization of ISF (external advisors or someone from within the organization).

Within the knowledge analysis, Wijers distinguishes two tasks: interpretation (leading to a text model) and conceptualization (leading to a conceptual meta model). The text model is an intermediate step between the verbal data and the final conceptual meta model (see Figure 6). In the next section, it will be shown that this distinction is not applied very strictly.

\subsection{Applied Methods}

An important step is the choice of a method of analysis. Due to a lack of experience in this area, it was decided to experiment with different methods by trying them out rather extensively. In this section, these methods and the experiences with each method will be summarized. Not every method was carried out for a whole experiment. Sometimes the disadvantages of certain methods became clear very soon. This 


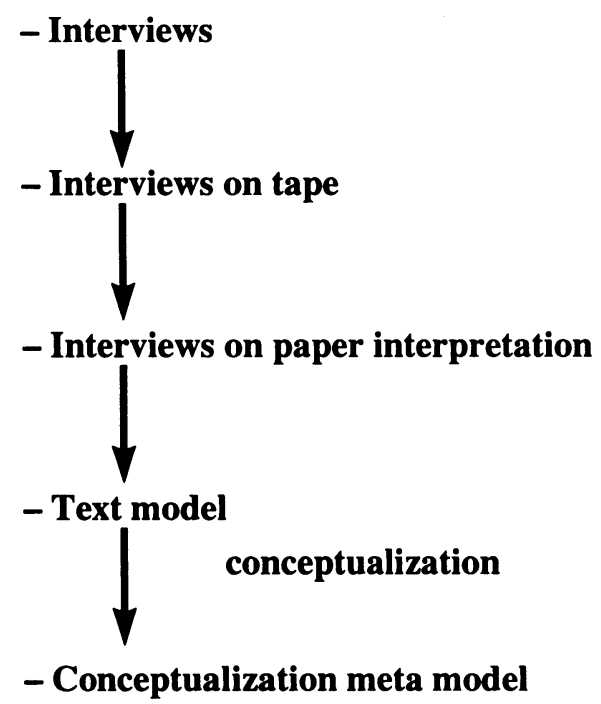

Figure 6 Knowledge Acquisition Tasks.

led to its immediate interruption. The original method was one that had been used before, within the Department of Information Systems at Delft University of Technology, to analyze expert knowledge in the area of information modeling (Wijers 1991; Verhoef 1993).

The use of the computerized tool Fragment. Fragment made it possible to perform both a task-oriented and a concept-oriented text analysis. The concept-oriented analysis meant that each text fragment had to be given a label, indicating of which concept this fragment was an instance and how it was related to other concepts. Concepts had to be chosen by the analyst. Fragment (a product of the Software Engineering Research Centre in the Netherlands) made it possible to generate reports with quantitative information on the protocols. This is a linear bottom-up approach that turned out to be rather labor intensive and it generated more pages of information than the 3,000 that formed the starting point for the analysis. The advantage of the approach was that it would afterwards be very simple to show that certain knowledge was indeed derived from the transcripts. Fragment does not support the creative step of determining what should be considered as a concept. Documenting instances of concepts took much more time than determining the concepts themselves. With a few thousand pages to go, very soon doubts came up as to the value added of the approach. 
Direct representation in diagrams. Just like the previously described method, this method aimed at representing the information from the transcriptions in diagrams. The construction of a text-based model as an intermediate step in the process of producing a formal representation using diagrams was skipped. It was decided to make three rounds through the transcript of each interview to come to a task structure, a concept structure, and an overview of the directives used in each interview. As a next step, these products had to be consolidated for the experiment as a whole. This approach has been applied for one experiment. During the analysis, task structures and concept structures were made by paper and pencil. After that, computerized tools were used to make diagrams out of them. The disadvantage of this approach for the construction of concept structures was the fact that the contents of a certain concept structure were influenced by the sequence in which the concepts were discovered. For each interview, this approach sometimes led to more than three handwritten pages with concepts and relations between them. A diagram was "finished" when, due to space limits, it turned out to be impossible to add an extra concept. This linear modeling approach prevented an optimal clustering of concepts. For the task structure, this problem turned out to be less serious.

Categorize and summarize first. A number of categories (i.e., ISF, information processing, internal environment of the ISF, external environment) were delineated that made it possible to classify each part of the text. Next to the classification, each logical unit of text was summarized. All information in one category was used as a basis for the concept structure of that category. Through combination and consolidation these separate structures led to one structure for the interview as a whole. Although it was now possible to construct consistent diagrams more readily, this method also ended up with very large and complex representations with lots of interrelations. Constructing them in an objective way and within a reasonable amount of time remained a problem. Influenced by the fact that quite a lot of energy already had been spent without satisfactory results, doubts began to increase as to the usefulness of the produced diagrams. Their usability in practice would be limited because of their complexity. Was it possible to attain the goal of the knowledge acquisition process without these complex diagrammatic representations of knowledge in task and concept structures? It was not very realistic to expect that it would be possible to end up with an algorithm that would automatically lead to the best solution for the ISF. The domain being dealt with concerned strategic decisions with a wide scope. An overly structured and formalized way of representation to support this type of decisions would not be possible. The methods tested so far might be useful as a basis for computerized knowledge based systems but were not a feasible solution in the area of organization of the ISF.

Textual representation of types of knowledge. From the conviction that the diagrammatic way of representation demanded much energy that was not compensated by profit in terms of insight, the choice was made to make a textual representation per knowledge category instead. The following eight knowledge categories were distinguished. 
- way of modeling: what does the expert want to know about an organization and its environment in order to get to know and to assess the organization, and in which terms does he express his view on the future organization of the ISF?

- way of diagnosis and design: how does the expert come to conclusions as to the present situation and how does he come to proposals for improvement?

- way of working: what activities does he perform in what sequence to come to a proposal for the design of the ISF?

- way of thinking: from which point of view does the expert act, what assumptions does he make?

- way of controlling: how does the expert manage the design process?

- way of supporting: what supporting tools does he use during the task execution?

- way of preparation: what does an expert know about the organization before he starts with the consultancy and what other knowledge turns out to be relevant during task execution?

- way of behavior: what else is important when working on the design of the ISF?

For the way of modeling, use was made of a categorization even more detailed than the one described in the previous approach. A linear approach was chosen to extract the information from the protocols. For each separate interview, the above knowledge categories were filled in. An incremental approach would have meant that from a certain interview only that information would be taken that was not covered by previously analyzed interviews. The advantage of the linear approach (which is more time consuming than an incremental approach) is that it would be possible in a later stage to make a comparison between different experts for each interview. The results of the analysis per interview for each expert were consolidated and restructured per knowledge category. This approach reduced the number of pages from more than 3,000 to about 450 .

Aggregation of the way of modeling in core topics. Because of the interdependence between certain knowledge categories, is was not easy to make a bottom-up analysis of an experiment. When describing the way of diagnoses and design, for instance, it was clear that use had to be made of the information stored under way of modeling, which consisted of a rather long list of nouns. It was decided to stop producing this list. From then on, certain core themes were distinguished and the description of each theme would cover both the information on the way of modeling and on the way of diagnosis and design; guidelines that directly referred to the design and analysis of the ISF were kept separate. This new approach automatically led to analysis reports that would be more readable for outsiders. To perform this last change, the results per interview of the previous method were used as a starting point. 


\subsection{Comparing the Results}

The individual reports (a total of 165 pages) of the nine experiments formed the basis of a mutual comparison, aiming at the discovery of correspondences between results of the nine analyzed experiments. Attention was also paid on gaining insight into the influence of the difference in type of organization upon the method followed by an expert and into the difference in methods applied by experts in one organization.

To allow for a better comparison of the way of design and analysis - guidelines directly regarding the ISF - they were categorized according to the contingency factors, design and goal parameters involved.

\section{SUMMARY OF THE RESULTS}

Although this paper focuses on the research approach, it is necessary for the assessment of this approach to summarize the research findings. The result (so far) of the research is phrased in terms of directives to be applied in practice for the design of the organization of ISF. As explained in section 2, the emphasis was on the acquisition of knowledge that the experts had in common. The summary of the results, in terms of guidelines, that is given in this section focus on that correspondence. It is not the goal of this paper, and it is impossible due to space limitations, to go into details of the results. The most important general conclusions will be given for each of the knowledge categories that are described in section 4.

For the subjects being most specific for the domain of the organization of the ISF (the way of thinking and the way of diagnosing and designing, as well as the way of modeling), this led to the following five main directives:

1. The design of the organization of the ISF has to take place from a dynamic and open system vision.

2. When organizing the vision, it has to be assumed that:

- responsibilities ought to be integral;

- changing the organization means changing information processing;

- organizing is a continuous, reactive and pro-active activity;

- the functioning of the organization depends more on people than on structure.

3. The vision of coordination ought to be that it is necessary in order to outweigh the disadvantages of self government.

4. Organizing ISF requires denoting goal variables, contingency factors and design variables. The most important contingency factors are the structure of the organization and the information related coherence between organization parts; the most important design variables are responsibilities, organizational place of the execution of activities and the procedure structure. 
5. The directives for the organization of the ISF can be summarized as follows: make line management responsible for the internal working and the external tuning, and integrate the task execution in the primary process.

The five directives (for demands to be made upon ISF designers) in the sphere of the way of preparation, way of supporting, way of thinking, way of working, and way of controlling are more general in character:

- Applying practical experience in the right way is essential; (fore)knowledge is a useful addition here.

- A framework (based on experience) for the problem field is the best way of supporting; a good memory is an important aid.

- Flexibility as to the way of acting is essential for the acceptance of the designer by the organization, and so for his effectiveness.

- The main line in the way of working is to be characterized as a top-down search for signals being connected with the organization of the ISF, where getting acquainted, analyzing and designing strongly overlap.

- Using appropriate techniques influences the productive use of the time available for intelligence.

\section{CONCLUSIONS}

The think aloud protocol method was used to elicit the expert knowledge. During the subsequent analysis, a considerable amount of time and effort was invested in experimenting with several techniques (first a technique known from literature was used but after that several changes were introduced before arriving at the final approach) for analyzing the protocols. On the one hand, there is the conviction that a detailed analysis is unavoidable when one tries to disclose all the information that is hidden in the protocols. However, when you go through the protocols several times, the awareness increases that someone else (another researcher or the same researcher at another point in time) would have missed certain information drawn from the protocols or would have discovered additional information; next to that, one is less objective when one goes through a protocol for the second time. On the other hand, the time that can be spent on the analysis is limited and the marginal benefits of another round through the protocols are uncertain and will certainly decrease. This makes this trade-off rather difficult. A group of masters' degree students were asked to analyze parts of the protocols. Based on these experiences, it can be concluded that it might have helped (in more than one way) to do the analysis in parallel with another researcher.

Because of the detailed analysis, a considerable amount of time was spent on rather general knowledge (i.e., how does the expert treat the interviewee). Nothing of the findings in this area have been published elsewhere. However, this perspective ought not to be excluded in a research project aimed at practical problem solving. The 
factors mentioned influence the acceptance of both the designer and his conclusions by the interviewees.

The use of diagrams to map the applied concepts and the performed tasks was very labor intensive because an attempt was made - like my colleagues who applied the same method - to end up with perfect diagrams. This diverted attention, for a period, from the actual use of the diagrams. In fact, they turned out to be too complex for practical usage. The exactness they suggested was another disadvantage. The opinion obtained here is that the required way to document the intermediate and final results is plain text. Text wise intermediate results can be used - certainly by someone who wrote them and who took part in the experiments - to compare the results of the nine experiments and to formulate final conclusions.

The comparison of the results of the individual experiments turned out to be a rather complex process. It required the active availability of a large amount of information. Another problem was that the bottom-up approach, which was characteristic for the way of analysis of the individual transcripts, was not the required approach for the cross protocol analysis. A noticeable fact is that in this research information which at first sight was incoherent and different working methods of experts could be organized in a relatively small number of coherent directives. On the basis of this result, further research can be directed toward the development of a methodology for the organization of the information (systems) function.

In this research project, a qualitative approach was used. No attention was paid to the number of times certain things occurred during the experiments. Even ex post this is not considered to be a disadvantage. The aim was not to determine in a statistically justified way how often certain ideas occur with certain experts. The aim was to try to document everything worthwhile for answering the research question even if it occurred only once in one of the nine experiments.

The importance of the guidelines is that they result from the comparison of the individual results of nine experiments: they are common knowledge. It was out of the scope of this research to verify the results of the knowledge acquisition process in practice. For the same reason, the cooperating companies were not asked to give their opinion about the research findings. Next to that, the only conclusion that could eventually have been drawn from such an experiment is that a company partly or fully agrees or disagrees with three experts.

The rather general nature of the research results might lead to the question whether knowledge engineering in the domain of the ISF yields anything specific and useful to practitioners. Based on the evaluation of a series of lectures presented on ISF design to IT professionals from Dutch organizations, the answer tends to be positive.

Working in close cooperation with three experts in three organizations was very interesting and offered the opportunity to learn more about the design of the IS function than it is possible to transfer to others. Next to that, especially the struggle through the knowledge analysis process, contributed a lot to self knowledge. 


\section{REFERENCES}

Butler Cox (1991). Managing the Devolution of Systems Responsibilities. Research Report 81, Butler Cox Foundation.

Bacon, C. J. (1990). “Organizational Principles of Systems Decentralization." Journal of Information Technology, Number 5, pp. 84-93.

Douma, S., and Schreuder, H. (1992). Economic Approaches to Organizations. Englewood Cliffs, New Jersey: Prentice Hall.

Mantelaers, P. A. H. M. (1995). Information Capacity Engineering. Doctoral Thesis (in Dutch), Delft University of Technology, Delft.

Swanson, E. B., and Beath, C. M. (1989). "Reconstructing the Systems Development Organization." MIS Quarterly, September, pp. 293-305.

Turner, W. S.; Langerhorst, R. P.; Hice, G. F.; Eilers, H. B.; and Uijttenbroek, A. A. (1988). System Development Methodology. Rijswijk, The Netherlands: Pandata B.V.

Verhoef, T. F. (1993). Effective Information Modeling Support. The Netherlands: Pijnacker.

Waterman, R.H., Jr.; Peters, T. J.; and Phillips, J. R. (1992). "The 7-S Framework." In H. Mintzberg and J. B. Quinn (Editors), The Strategy Process. Englewood Cliffs, New Jersey: Prentice-Hall.

Wijers, G. M. (1991). Modeling Support in Information Systems Development. Amsterdam: Thesis Publishers.

\section{BIOGRAPHY}

Paul Mantelaers is associate professor of information systems at Delft University of Technology, Department of Information Systems. After his graduation in 1974 from Tilburg University, he worked at the Dutch Central Bureau of Statistics, first as a national accounts statistician, later in the area of information systems design. In 1984, he joined Delft University of Technology and received his Ph.D. in 1995. He teaches information management and development of information systems. His principal research focus is on the design of the IS function and on the development and management of transnational information systems. 